\section{Polymer Photodetector Developed with High Detectivity Across Broad Spectral Range}

Photodetectors sensitive to the full UVvisible to near-infrared spectrum are very desirable for scientific and technological applications, but conventional semiconductor detectors are limited to narrower subbands of the spectrum. Full coverage thus requires separate sensors for different subbands, which is a complicated and costly solution at best. As published in the September 25 issue of Science (DOI: 10.1126 /science.1176706; p. 1665), a group of researchers have addressed this problem with a new polymer photodetector (PPD) with broad spectral response and unusually high detectivity. $X$. Gong and A.J. Heeger from the University of California in Santa Barbara and CBrite Inc., Y. Cao from South China University of Technology, and their colleagues have built a PPD that delivers a response from $300 \mathrm{~nm}$ to $1450 \mathrm{~nm}$ in a device that incorporates a small bandgap conjugated polymer with a fullerenederivative in the active region.

Gong and co-workers fabricated their
PPD by spin-casting a mixture of poly $(5,7-$ bis(4-decanyl-2-thienyl)-thieno (3,4- $b$ ) diathiazole-thiophene-2,5) (PDDTT) and $(6,6)$-phenyl- $C_{6} 1$-butyric acid methyl ester $\left(\mathrm{PC}_{60} \mathrm{BM}\right)$ between an $\mathrm{InSnO}_{3}$ (ITO) anode and $\mathrm{Al}$ cathode. The PDDTT:PC ${ }_{60} \mathrm{BM}$ forms an interpenetrating donor and acceptor bulk heterojunction network in the active layer, allowing efficient photogeneration and collection of carriers. The researchers said that shot noise from dark current limits detectivity and, under this assumption, attempt to maximize detectivity by incorporating additional materials in a multilayer geometry to block or suppress dark current. They fabricated a number of devices including layers of various polymers and fullerene-derivatives, and measured the photocurrent and dark current of each device at $-100 \mathrm{mV}$ bias. From these data they calculated the detectivity and found the best response from devices with layers of polystyrene-N, N-diphenyl-N,N-bis(4-n-butylphenyl)(1,10-biphenyl)-4,4-diamine-perfluorocyclobutane (PS-TPB-PFCB) and $\mathrm{C}_{60}$. The layering order of this device was ITO/PEDOT / PS-TPD-PFCB / PDDTT:
$\mathrm{PC}_{60} \mathrm{BM} / \mathrm{C}_{60} / \mathrm{Al}$. The $\mathrm{C}_{60}$ layer prevents photogenerated holes from reaching the Al cathode and the PS-TPD-PFCB blocks electrons from the ITO anode, resulting in a dramatically decreased dark current. PEDOT is poly $(3,4$-ethylenedioxy-thiophene).

For incident wavelengths of $300 \mathrm{~nm}$ to $1450 \mathrm{~nm}$ the calculated detectivity of this device is near or above that of $\mathrm{Si}$ and InGaAs detectors, ranging from $10^{14}$ Jones to $10^{12}$ Jones (where Jones $=\mathrm{cm} \mathrm{Hz}^{1 / 2} / \mathrm{W}$ ). A further advantage of the PPD over InGaAs is that this impressive detectivity is achieved at room temperature, whereas InGaAs photodetectors must be cooled to near $4.2 \mathrm{~K}$ to achieve this detectivity. The researchers also demonstrated that the PPD has a linear dynamic range much larger than InGaAs photodetectors and almost as large as Si photodetectors. Given the favorable comparison to conventional photodetectors and the simple, inexpensive fabrication technique, Gong and coworkers are optimistic that their polymer photodetector will prove valuable to a variety of applications.

AlisOn HatT

\section{Pinning Down Superconductivity to a Single Layer}

Using precision techniques for making superconducting thin films layer-by-layer, G. Logvenov, A. Gozar, and I. Božović of Brookhaven National Laboratory have identified a single layer responsible for one such material's ability to become superconducting. The technique, described in the October 30 issue of Science (DOI: 10.1126/science.1178863; p. 699), could be used to engineer ultrathin films with "tunable" superconductivity for higher efficiency electronic devices.

"We wanted to answer a fundamental question about such films," said physicist and group leader Ivan Božović. "Namely: How thin can the film be and still retain high-temperature superconductivity?"

The thinner the material (and the higher its transition temperature to a superconductor), the greater its potential for applications where the superconductivity can be controlled by an external electric field. "This type of control is difficult to achieve with thicker films, because an electric field does not penetrate into metals more than a nanometer or so," Božović said.

To explore the limits of thinness, Božović's group synthesized a series of films based on the high-temperature superconducting cuprates (copper-oxides) - materials that carry current with no energy loss when cooled below a certain transition temperature. Since zinc is known to suppress the superconductivity in these materials, the scientists systematically substituted a small amount of zinc into each of the copper-oxide layers. Any layer where the zinc's presence had a suppressing effect would be clearly identified as essential to superconductivity in the film.

"Our measurements showed that the zinc doping had essentially no effect, except when placed in a single, well-defined layer. When the zinc was in that layer, the superconductivity was dramatically suppressed," Božović said.

The material studied by Božović's team was unusual in that it consists of layers of two materials, one metallic and one insulat-

\section{$\mathrm{J} A \mathrm{~N}$ IS}

Cryogenic Systems

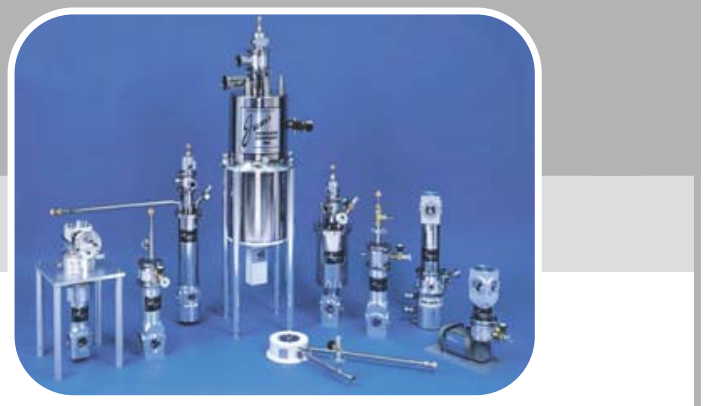

Does your research require low temperatures? Contact Janis today. Our engineers will assist you in choosing the best system for your application.
$10 \mathrm{mK}$ to $800 \mathrm{~K}$
Cryocoolers
Magnet Systems
LHe/LN ${ }_{2}$ Cryostats
Dilution Refrigerator Systems
Janis Research Company
2 Jewel Drive Wilmington, MA 01887 USA
TEL +1978 657-8750 FAX +1978 658-0349 sales@janis.com
Visit our website at WWW.janis.com 